\title{
Chitosan/aminoacid hydrogels with antimicrobial and bioactive properties as new scaffolds for human mesenchymal stem cells culture applicable in wound healing
}

\author{
M. Piatkowski ${ }^{*}$, D. Kitala ${ }^{2}$, J. Radwan-Pragłowska ${ }^{1}$, L. Janus $^{1}$, A. Klama-Baryła ${ }^{2}$, W. Łabus' ${ }^{2}$, \\ E. Tomanek2, J. Glik²,3, D. Matýsek ${ }^{5}$, M. Kawecki ${ }^{2,4}$ \\ ${ }^{1}$ Department of Biotechnology and Physical Chemistry, Faculty of Chemical Engineering and Technology, Cracow \\ University of Technology, Warszawska 24 St, 31-155 Cracow, Poland \\ ${ }^{2}$ Dr Stanislaw Sakiel Center for Burns Treatment, Siemianowice Slaskie, Poland \\ ${ }^{3}$ Department of Chronic Wounds Management Organization, School of Health Science in Katowice, Medical University \\ of Silesia, Katowice, Poland \\ ${ }^{4}$ Department of Health Sciences, Technical-Humanistic Academy, Bielsko-Biala, Poland \\ ${ }^{5}$ Faculty of Mining and Geology, Technical University of Ostrava, 17. Listopadu 15 St, Ostrava-Poruba, Czech Republic
}

Received 16 Juny 2017; accepted in revised form 17 September 2017

\begin{abstract}
Application of human mesenchymal stem cells brings a new hope for advanced wound healing. To enable cells culture and their viability maintenance, a new type of biomaterials must be developed. Scaffolds prepared from polymers of natural origin can mimic extracellular matrix. Chitosan, which is a chitin derivative, has many favorable properties like biodegradability or lack of cytotoxicity. Therefore, it is widely applied in medicine and pharmacy. Nevertheless, its chemical modification may lead to the loss of biocompatibility of the material and generate some significant problems with cells culture. In this article a novel strategy of the bioactive and antimicrobial chitosan hydrogel scaffolds is presented. As crosslinking agents non-toxic substances were used such as aspartic acid and glutamic acid. Obtained biomaterials were investigated over their chemical structure, morphology and biological activity. Performed tests using human mesenchymal stem cells confirmed bioactivity and biocompatibility, as well as antibacterial and antifungal properties.
\end{abstract}

Keywords: biocompatible polymers, hydrogel biomaterials, scaffolds, stem cells, chitosan

\section{Introduction}

Rapid development of tissue engineering and regenerative medicine raised a need for the preparation of new type of biomaterials. Special attention of scientists is focused on polymers and biopolymers, which can create different porous structures while maintaining their biocompatibility.

Cellular therapies exhibit high potential for repairing damaged tissues [1], and thus it is thought that stem cell injections are a promising treatment method within the scope of regenerative medicine $[2,3]$. Mesenchymal amniotic stem cells became applicable in tissue regeneration or even nerve injury repair [4]. The injection method of stem cell delivery was a serious limitation [2]. Biomaterials are characterized by the ability to maintain MSC (mesenchymal stem cell) functionality, which can translate into improvement of the quality of cell therapies. It was demonstrated that agarose-carbomer hydrogel positively affects the number of live cells. In the same study, biomimetic hydrogel was able to significantly immunomodulate inflammation [5]. Hydrogel biomaterials ensure even distribution of cells in the material and their high viability [6]. Hydrogel scaffolds

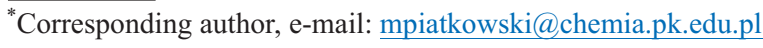
(C) BME-PT 
provide the opportunity to encapsulate stem cells while maintaining their viability [7]. They remain live and metabolically active for up to 7 days [8]. It is believed that composite hydrogels are suitable for controlling stem cell differentiation and bone tissue regeneration [9]. Literature data show that hydrogels form a three dimensional microenvironment for stem cells precisely mimicking in vivo conditions, which leads to stimulation of cellular proliferation and differentiation [10]. Among other uses, biomaterials are applied in improving low solubility of medicines in water and their chemical stability as well as overcoming the skin barrier [11]. Structural properties of hydrogel depend mainly on the material used in its construction and the way it is manufactured [7]. However, most hydrogel dressings are formed using UV cross-linking or require multi-stage modification based on chemical reactions and purification methods, which causes safety problems, increases costs and requires complicated production methods [12]. Some of the most significant parameters when designing a hydrogel scaffold are swelling, mechanical properties and material degradation in time, which are strictly correlated with the cross-linked structure of hydrogel being controlled by various manufacturing conditions [11]. The hydrogel scaffolds that are currently available have many advantages but obtaining the required mechanical properties still constitutes a challenge [1]. Many polymers used in the production of hydrogel are natural, e.g. alginate, collagen, fibrin, chitosan as well as gelatin or hyaluronan. These polymers have the advantage of native biocompatibility and presence of natural binding sites, which provide effective interaction between cells and hydrogels. However, low stability, poor mechanical properties and quick degradation are the main drawbacks of natural hydrogels. Chitosan is a derivative of chitin obtained during the deacetylation process. It consists of $N$-acetylaminoglucose and aminoglucosemers joined by glycosidic bonds [15]. For many years, it has been widely used in medicine delivery and tissue engineering due to its high biocompatibility, low toxicity and mechanical properties that can be adjusted to natural ECM $[11,13]$. In the last decade, a series of studies have been conducted regarding chitosan hydrogels, mostly in the form of injections. However, the main obstacle for hydrogel injection is the gelation time - if gelation takes place too quickly the needle gets blocked, while in the case of slow gelation cells, growth factors or medicines are lost [11].
Additionally chemical modification of chitosan in order to increase its durability may lead to the loss of biocompatibility. Moreover, during chitosan scaffolds obtainment very often toxic crosslinking agents are applied, such as 1-ethyl-3-(3-dimethylaminopropyl)carbodiimide (EDS), which may generate negative effects resulting in inflammation or fibrosis [16]. Other substances used for hydrogels preparation like genipin and glutaraldehyde may exhibit neurotoxic effects and significantly reduce hydrophilicity and adhesiveness [17-20].

In this article a new type of chitosan-based hydrogel scaffolds are presented. Chitosan hydrogels were obtained using non-toxic and bioinert/bioactive crosslinking agents - aspartic acid (Asp), glutamic acid (Glu) and glycerol, under microwave radiation. Ready biomaterials were investigated for their biological properties like antibacterial and antifungal activity, as well as lack of cytotoxicity. Moreover, bioactivity of chitosan scaffolds using human mesenchymal stem cells was evaluated.

\section{Experimental}

\subsection{Materials}

All chitosan hydrogels were synthesized using chitosan with viscosity average molar mass $410000 \mathrm{~g} / \mathrm{mol}$ and $80 \%$ deacetylation degree, purchased from Vanson, Redmond, USA. Glutamic acid, aspartic acid and glycerol were purchased from Sigma Aldrich, USA. No modifications of the materials were performed before the synthesis.

\subsection{Microwave-assisted chitosan hydrogels synthesis}

For the synthesis of chitosan hydrogels $1.0 \mathrm{~g}$ of biopolymer was dissolved in $10 \mathrm{~cm}^{3}$ of $2 \%$ acetic acid solution, then different amounts of aminoacids and $3 \mathrm{~cm}^{3}$ of glycerol, were added. Ready reaction mixtures were placed in Prolabo Synthewave 402

Table 1. Hydrogels synthesis parameters

\begin{tabular}{|c|c|c|c|}
\hline Sample & $\begin{array}{c}\text { Asp:Glu:chitosan ratio } \\
{[\mathbf{g}]}\end{array}$ & $\begin{array}{c}\text { Power } \\
{[\mathbf{W}]}\end{array}$ & $\begin{array}{c}\text { Temperature } \\
{\left[{ }^{\circ} \mathbf{C}\right]}\end{array}$ \\
\hline J1 & $0.1: 0: 1.0$ & 300 & 150 \\
\hline J2 & $0: 0.1: 1.0$ & 300 & 150 \\
\hline J3 & $0.1: 0.1: 1.0$ & 400 & 180 \\
\hline J4 & $0.2: 0.1: 1.0$ & 400 & 180 \\
\hline J5 & $0.1: 0.2: 1.0$ & 500 & 180 \\
\hline J6 & $0.2: 0.2: 1.0$ & 600 & 200 \\
\hline J7 & $0.2: 0.2: 1.0$ & 500 & 180 \\
\hline
\end{tabular}


microwave reactor and irradiated for $30 \mathrm{~min}$. Microwave-assisted chitosan hydrogels synthesis parameters are presented in Table 1.

\subsection{Fourier transform infrared spectroscopy (FT-IR) analysis}

FT-IR analysis was performed using IR Nicolet 6700 spectrometer, USA in the range between 400 and $4000 \mathrm{~cm}^{-1}$. Dried hydrogels samples were placed in $\mathrm{KBr}$ pellets. For each analysis $200 \mathrm{mg}$ of $\mathrm{KBr}$ was used and $5 \mathrm{mg}$ of analysed sample. The resolution was $4 \mathrm{~cm}^{-1}$, number of scans was 32 .

\subsection{Scanning electron microscope (SEM) analysis}

SEM analysis was performed using FEI QUANTA 650 FEG. All samples before the analysis were lyophilized. Microphotographs were taken under pressure of $50 \mathrm{~Pa}$ and $\mathrm{HV}$ of $20.00 \mathrm{kV}$.

\subsection{Incubation study}

\subsubsection{Incubation study in simulated body fluid (SBF)}

All reagents used for preparation of $1 \mathrm{dm}^{3}$ of SBF are listed in Table 2. $\mathrm{pH}$ value of the tested solutions was measured after 0, 1, 24, 48, 144 and 192 hours. For the $\mathrm{pH}$ measurements, $\mathrm{pH}$-sensitive electrode Hydromet ERH-11 (Poland) was used and between measurements all tested solutions were incubated at $37^{\circ} \mathrm{C}$ under sterile conditions. For the tests $1.0 \mathrm{~g}$ of dried hydrogel was used. The test was repeated five times. As a reference distilled water was used.

\subsubsection{Incubation study in Ringer's Fluid}

All reagents used for preparation of $1 \mathrm{dm}^{3}$ of Ringer's Fluid are listed in Table 3. $\mathrm{pH}$ value of the tested solutions was measured after $0,1,24,48,144$ and 192 hours. For the $\mathrm{pH}$ measurements, $\mathrm{pH}$-sensitive electrode Hydromet ERH-11 (Poland) was used and

Table 2. SBF composition

\begin{tabular}{|c|c|}
\hline Chemical reagent & $\begin{array}{c}\text { Amount } \\
\text { [g] }\end{array}$ \\
\hline $\mathrm{NaCl}$ & 7.996 \\
\hline $\mathrm{NaHCO}_{3}$ & 0.350 \\
\hline $\mathrm{KCl}$ & 0.224 \\
\hline $\mathrm{KH}_{2} \mathrm{PO}_{4} \cdot 3 \mathrm{H}_{2} \mathrm{O}$ & 0.228 \\
\hline $\mathrm{MgCl}_{2} \cdot 6 \mathrm{H}_{2} \mathrm{O}$ & 0.305 \\
\hline $\mathrm{CaCl}_{2}$ & 0.278 \\
\hline $\mathrm{Na}_{2} \mathrm{SO}_{4}$ & 0.071 \\
\hline $\mathrm{HCl}$ & Until $\mathrm{pH}=7.25$ \\
\hline
\end{tabular}

between measurements all tested solutions were incubated at $37{ }^{\circ} \mathrm{C}$ under sterile conditions. For the measurements $1.0 \mathrm{~g}$ of dried hydrogel was used. The test was repeated for five times. As a control distilled water was used.

\subsection{Isolation and culture of human amnion mesenchymal stem cells}

Cell and Tissue in vitro Culture Laboratory with Tissue Bank has obtained the approval of the Ministry of Health for isolation, processing and storage of amnion mesenchymal cells and the approval of the Main Pharmaceutical Inspectorate for advanced therapy medicinal product manufacturing. The placenta was obtained during Caesarean section. The qualification examinations of the live donors included: HIV-1 and 2 (Anti-HIV-1 and 2), hepatitis B (HBsAg and Anti$\mathrm{HBc}$ ), hepatitis $\mathrm{C}$ (Anti-HCV) and a specific test for syphilis. The culture was conducted in class B Clean Rooms under laminar flow hood (class A; HSKS 18, Thermo). In order to isolate cells from human amnion, a tissue material fragment of about $20 \mathrm{~cm}^{2}$ was used. The cells were isolated using a mechanical homogenisation method according to our previously described protocol [13] and the culture was conducted using the medium AmnioGrow (Cytogen). The material sterility was tested by sending a tissue fragment for microbiological analysis performed according to our previously described standard [14]. The primary culture was conducted for 14 days. The culture was conducted in $37^{\circ} \mathrm{C}, 95 \%$ humidity and $5 \%$ $\mathrm{CO}_{2}$ (incubator Heracell 240; Thermo Fisher Scientific) until reaching $80 \%$ confluence and then etched (using TrypLETM Select (1X) solution, no Phenol Red, Thermo Fisher Scientific) and stem cells were isolated through positive selection by the surface antigen CD105. The MACS columns were placed in a MACS separator (MiltenyiBiotec) providing magnetic field necessary for keeping the labelled cells. After the column isolation, the cells were evaluated to check whether they met the minimum criteria for stem cells. Their adherence to plastic was examined through microscopic observation using an Olympus IX81 motorised inverted microscope. The analysis

Table 3. Ringer's Fluid composition

\begin{tabular}{|c|c|}
\hline Chemical reagent & $\begin{array}{c}\text { Amount } \\
\text { [g] }\end{array}$ \\
\hline $\mathrm{KCl}$ & 8.60 \\
\hline $\mathrm{NaCl}$ & 0.30 \\
\hline $\mathrm{CaCl}_{2} \cdot 6 \mathrm{H}_{2} \mathrm{O}$ & 0.48 \\
\hline
\end{tabular}


of differentiation into 3 mesodermal cell lines was performed using Human Mesenchymal Stem Cell Functional Identification Kit (R\&D Systems). After positive verification, the cells were used in further studies.

\subsection{The evaluation of stability of culture mediums after sterilization}

Two methods of medium sterilisation were selected: ethylene oxide and steam $120-134^{\circ} \mathrm{C}$. Overpressure steam sterilisation was performed using a compact steam steriliser Sterivap Unisteri from BMT MMM Group. The sterilising factor used in the procedure was overpressure saturated steam in the temperature range of $120-134^{\circ} \mathrm{C}$. The steam sterilisation parameters i.e. temperature, pressure and time were adjusted to the tested products by a series of sterilisation attempts allowing for optimisation of the tested material sterilisation method. The sterilisation procedure was conducted according to the manufacturer's manual. The evaluation of the tested material sterilisation process efficiency was performed on the basis of the device sterilisation process print-out as well as biological and chemical tests of a given sterilisation pack cycle.

Ethylene oxide sterilisation was performed using the 'Steri-Vac 5XLP' gas steriliser/aerator from 3M. In the course of this process, the tested material was subjected to gas sterilisation using ethylene oxide in a tightly sealed chamber. Ethylene oxide is a very efficient sterilising factor but, due to its toxicity, the tested material had to be subjected to degassing (aeration). The whole process was performed in a fully automated steriliser. The total sterilisation cycle time including degassing was 16 hours and the sterilisation alone took 4 hours. The sterilisation was conducted using Steri-GasTM, 4-100, 100 g cartridges from $3 \mathrm{M}$ containing ethylene oxide (EO) as active substance, $100 \mathrm{~g}$ per $100 \mathrm{~g}$ of the product (N.i. 603023-00-X). The sterilisation process was performed according to the manufacturer's manual of the 'SteriVac 5XLP' gas steriliser/aerator from 3M. The confirmation of the sterilisation process correctness was made by checking the print-out from the steriliser printer including information about the course of the whole sterilisation cycle.

\subsection{The evaluation of culture medium stability}

Because the culture media are to be used for culturing and co-culturing, it should be assumed that it is
Table 4. Density equivalents in McFarland units and optical density

\begin{tabular}{|c|c|}
\hline Standard McFarland scale & $\begin{array}{c}\text { Theoretical optical densities } \\
\text { for } \mathbf{5 5 0} \mathbf{~ n m}\end{array}$ \\
\hline 0.5 & 0.125 \\
\hline 1.0 & 0.25 \\
\hline 2.0 & 0.50 \\
\hline 3.0 & 0.75 \\
\hline
\end{tabular}

necessary to keep them in cultures dedicated to specific cell lines in the following conditions: $37^{\circ} \mathrm{C}$, $95 \%$ humidity and $5 \% \mathrm{CO}_{2}$. Samples J3, J4, J5, J7 were divided into 3 parts and placed in a six-hole plate (Starsted) and had $5 \mathrm{~mL}$ of culture nutrients poured over them: AmnioGrow (Cytogen), DMEM (Biosera) $+10 \%$ FBS (Biosera) and KSFM (Thermo Fisher Scientific). Photographic documentation was prepared using a CANON EOS 450D camera after 2 and $24 \mathrm{~h}$ after nutrient application. Additionally, the amount of the medium 'absorbed' by the scaffold was evaluated. In order to do that, after $24 \mathrm{~h}$, the remaining amount of the medium was collected from the six-hole plate using an automatic pipette of $1-5 \mathrm{~mL}$ volume (Eppendorf). Turbidity analysis was also conducted depending on the type of medium and culture medium using a DENSICHEK device intended for measurement of optical density of suspensions placed in a test tube with liquid medium. It provides results in McFarland units (Table 4). Polystyrene test tubes from BioMerieux were used in the procedure (ref. 69285). The device has the following optical characteristics: light source LED $590 \mathrm{~nm}$, logarithmic display in McFarland units $0-4.5 \mathrm{McF}$, the reading accuracy is $\pm 0.10 \mathrm{McF}$. The procedure utilised density equivalents in McFarland units and optical density with the following parameters.

Before measurement, the device calibration was verified using a standard calibration test tube (ref. 93059). The obtained solutions were placed in test tubes and their density was subsequently measured using McFarland scale.

\subsection{The evaluation of sterility after the production process}

Before and after the sterilisation process, the samples were evaluated for their colonisation with microorganisms according to our previously described procedure [14]. The material samples were fully immersed in the medium. Aerobic bacteria were cultured in liquid medium Thioglycollate Bulion USP (Oxoid), while fungi and anaerobic bacteria were 
cultured in casein soya broth (TryptonaSoya Bulion USP, Oxoid). The culture was conducted for 7 days.

\subsection{Standardisation of attaching media to the bottom of the bottle}

In order to perform effective and standardised plating the medium must be attached to the bottom of a multi-hole plate. Foetal bovine serum (FBS: Biosera) was used in the amount of 25,50 and $100 \mu \mathrm{L}$ per hole (12-hole plate) in order to attach fragments after sterilisation to the bottom of culture plate. Photographic documentation was prepared and the plate was rotated by $180^{\circ}$ (upside down) to check the efficiency of scaffold attachment to the culturing dish.

\subsection{Impact on population doubling in stem cell culture}

The cells were plated onto a multi-hole plate (Sarsted) containing media. Population doubling in the cultures was examined using a Tali ${ }^{\circledR}$ Image-Based Cytometer (Life Technologies). The analysis was performed according to the manufacturer's protocol. The number of cells was evaluated at the moment of plating and at culture termination. Population doubling was calculated using Equation (1):

$$
\mathrm{PT}=\frac{\left(\log N_{\mathrm{h}}-\log N_{0}\right)}{\log (2)}
$$

where $N_{\mathrm{h}}$ number of cells on the day of culture termination, $N_{0}$ number of plated cells.

Photographic documentation was also prepared in order to verify whether contact growth inhibition occurred.

\subsection{Plating the cultured stem cells on the scaffolds}

$4.5 \cdot 10^{5}$ mesenchymal stem cells isolated from human amnion were plated on the scaffolds in the top right corner. Next, $1 \mathrm{~mL}$ of AmnioGrow culture nutrient was added to each culture. The cells were cultured for one week and the culture nutrient was changed every $48 \mathrm{~h}$. The samples were fixed with $1 \mathrm{~mL} \mathrm{3.7 \%} \mathrm{formaldehyde} \mathrm{and} \mathrm{examined} \mathrm{using} \mathrm{the}$ classical haematoxylin and eosin staining.

\section{Results and discussion}

\subsection{Hydrogel scaffolds synthesis}

As a result of microwave-assisted synthesis four hydrogels were obtained. In the case of samples J1 and $\mathrm{J} 2$ the crosslinking process did not occur due to insufficient amount of crosslinking agents and too low temperature of the process. In the case of samples J3, J4, J5 and J7 functional gels with the capability of water sorption were synthesized. Sample J6 underwent thermal degradation due to too high temperature of process, as well as irradiation power. Samples $\mathrm{J} 3$, J4, J5 and J7 were used for further experiments.

\subsection{Incubation study}

\subsubsection{Incubation study in SBF}

All four samples were of similar behavior in SBF (Figure 1). During first 92 hours small increase in $\mathrm{pH}$ value was observed which could be associated with the protonation of free $\mathrm{NH}_{2}$ groups. After 92 hours the $\mathrm{pH}$ value was constant and the incubation solution was in equilibrium state. No significant $\mathrm{pH}$ changes suggests that the chemical composition of hydrogel scaffolds in SBF medium is stable and the biomaterials do not react with the components of body fluid.

\subsubsection{Incubation study in Ringer's Fluid}

Results of incubation study performed in Ringer's Fluid gave similar results to those performed in SBF (Figure 2). All four samples were of similar behavior.

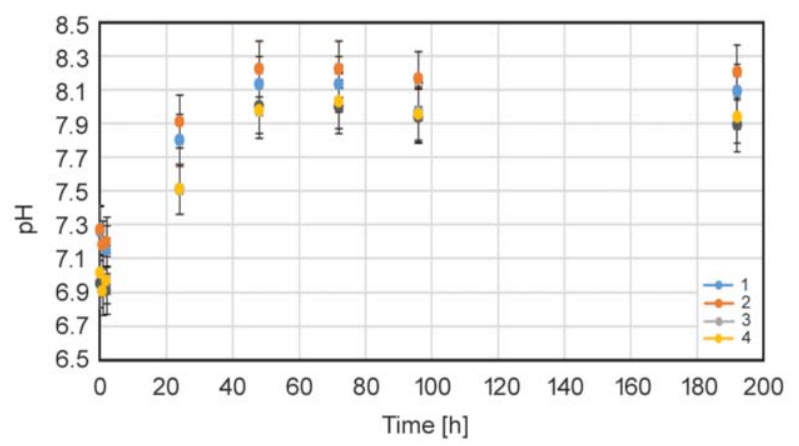

Figure 1. Incubation study of hydrogel scaffolds in SBF: 1 - J3 sample, 2 - J4 sample, 3 - J5 sample, 4 - J5 sample

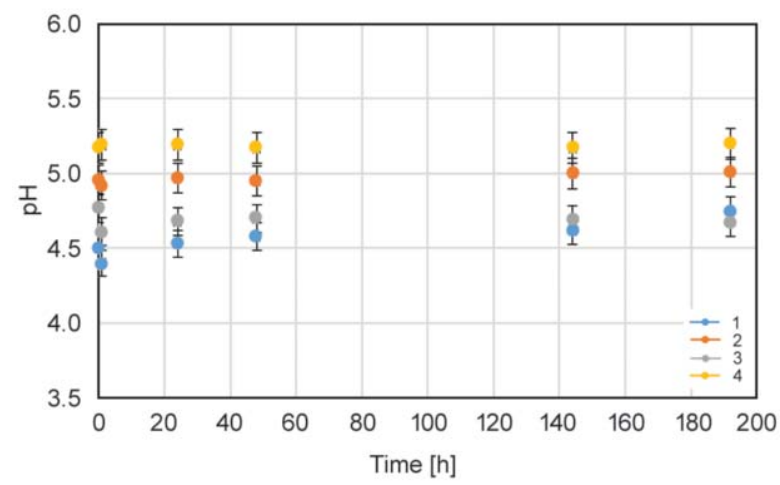

Figure 2. Incubation study of hydrogel scaffolds in Ringer's Fluid; 1 - J3 sample, 2 - J4 sample, 3 - J5 sample, 4 - J7 sample 
During first 48 hours a very slight $\mathrm{pH}$ value increase was observed which could be correlated with the protonation of $\mathrm{NH}_{2}$ groups. After 48 hours the $\mathrm{pH}$ value was constant and the incubation solution was in equilibrium state. No $\mathrm{pH}$ changes suggests that the chemical composition of hydrogel scaffolds in Ringer's fluid is stable and the biomaterials do not react with the components of this medium.

It can be stated that hydrogels were inert to both incubation media, what suggests that hydrogel scaffolds do not undergo biodegradation process without the presence of microorganisms or appropriate human enzymes. The hydrogels are durable in SBF and Ringer's fluid and do not react with their ingredients. Therefore, the possibility of the formation of toxic

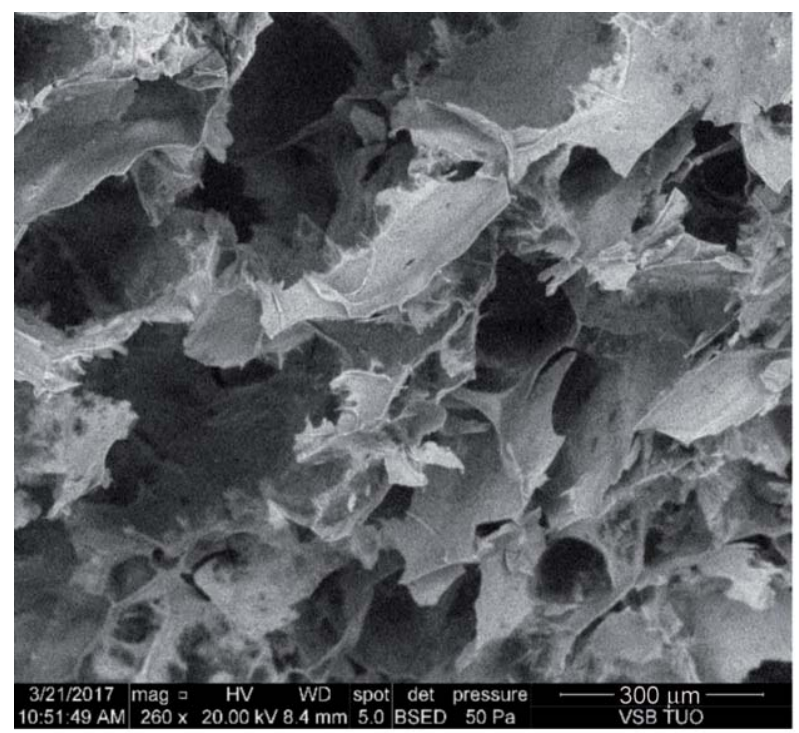

a)

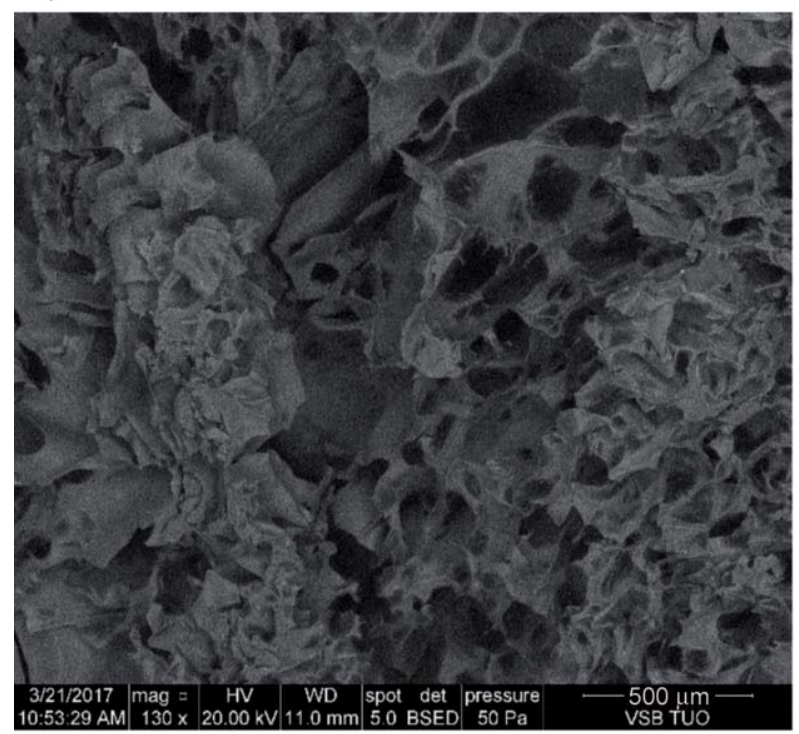

c) degradation products or products of chemical reactions with incubation solutions can be eliminated.

\subsection{SEM analysis}

In the case of all samples high porosity of the materials can be observed (Figure 3). Also strong correlation between the quantity of crosslinking agents used during the synthesis and morphological structure can be noticed. The most uniform and the smallest pores are observed in the case of sample J7. All samples have porous structure, thus it can be assumed that they will enable oxygen and nutrients, as well as growth factors delivery. Additionally, formed channels may enable cells adhesion, migration and proliferation. Also biomaterials seem to ensure good

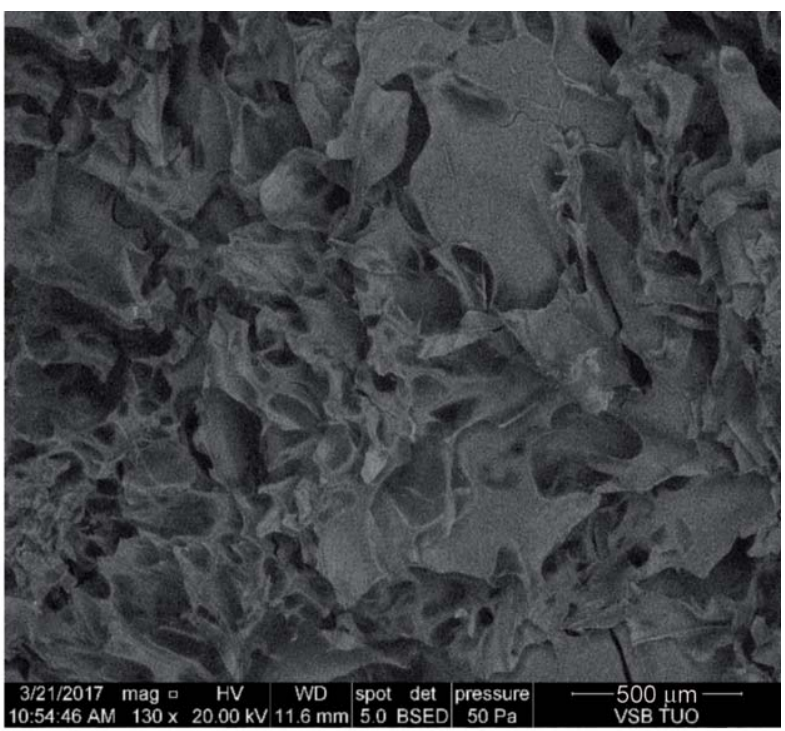

b)

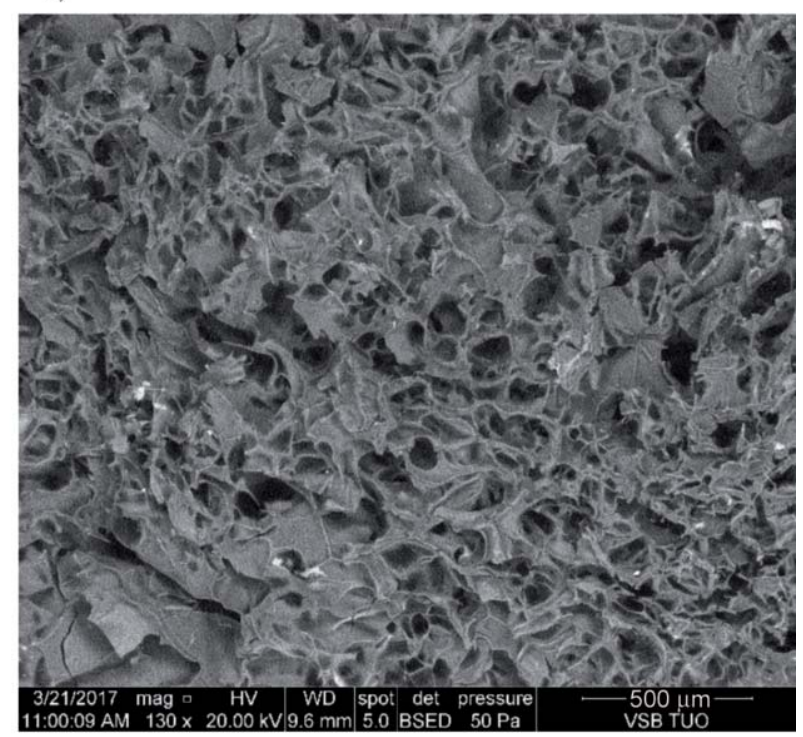

Figure 3. SEM microphotographs of hydrogel scaffolds: (a) J3 sample, (b) J4 sample, (c) J5 sample, (d) J7 sample 
Table 5. Macroscopic change in the appearance of the samples sterilised using various methods

\begin{tabular}{|c|c|c|}
\hline Appearance befor sterilization & After sterilization with ethylene oxide & After sterilization with steam \\
\hline 1 & & \\
\hline
\end{tabular}

conditions for neovascularization process occurrence. It can be stated that sample $\mathrm{J} 7$ is the most appropriate in the case of pores structure due to their size, shape and distribution. Thus it may accelerate differentiation process of the Mesenchymal Stem Cells (MSC). The size of pores can be strongly correlated with sorption capacity of the obtained hydrogels. SEM microphotographs demonstrate that the pores size, shape and distribution can be adjusted to special requirements by choosing the most appropriate amount of aminoacids. Figure 4 also shows that the most porous structure is obtained in the case of two types of aminoacids application for the crosslinking process.

\subsection{Evaluation of medium stability after sterilisation}

The samples were slightly deformed, which did not negatively affect the possibility to use them in cultures (Table 5).

\subsection{FT-IR analysis}

Figure 4 demonstrates FT-IR spectra of pure chitosan (Figure 4a), obtained J7 sample before (Figure 4b)

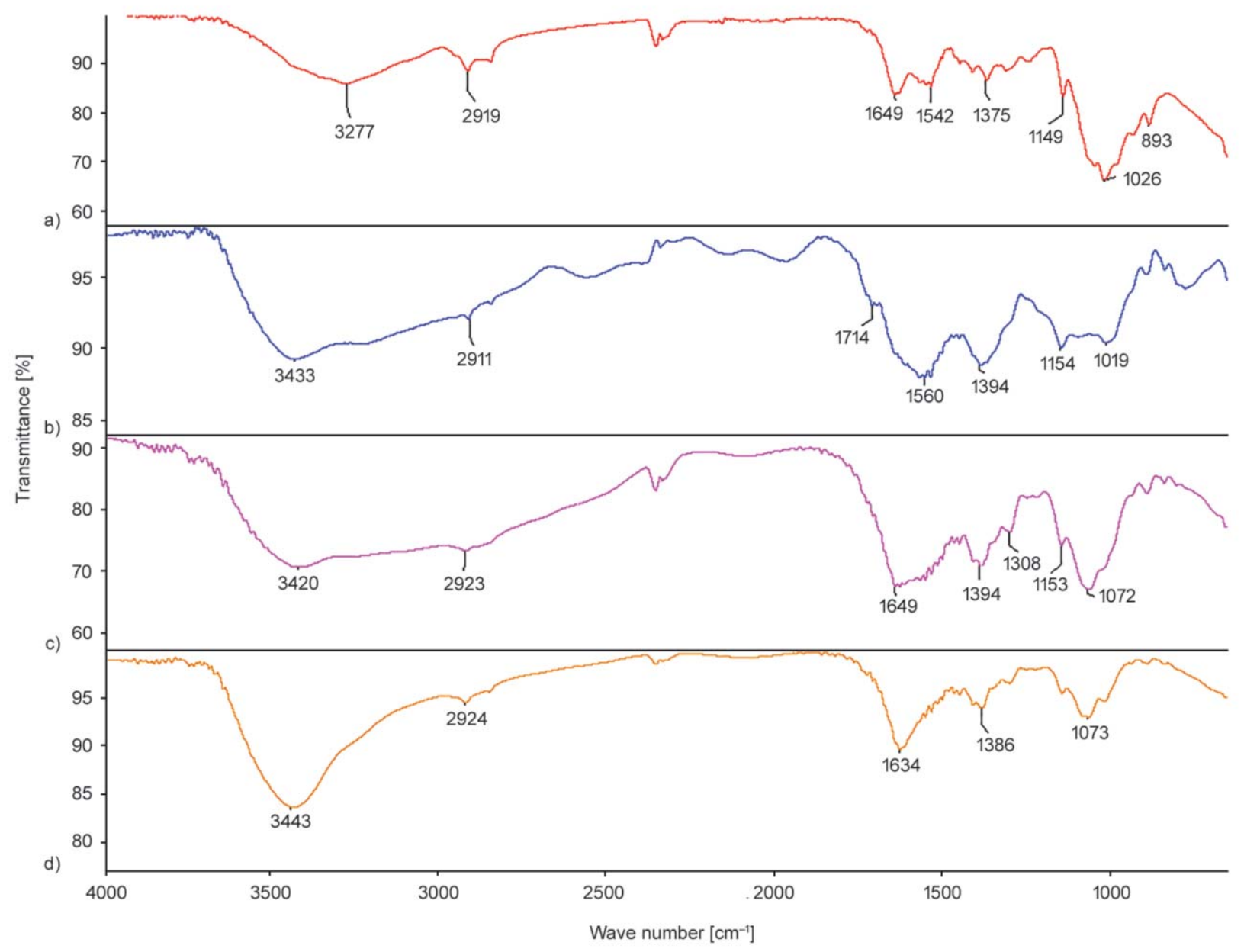

Figure 4. FT-IR spectra of pure chitosan (a) and J7 sample: before sterilization (b), after sterilization with water vapor (c), after sterilization with ethylene oxide (d) 
and after sterilization process with water vapor (Figure $4 \mathrm{c}$ ) and ethylene oxide (Figure 4d). In the spectrum of pure chitosan band with the maximum at $3277 \mathrm{~cm}^{-1}$ corresponds with stretching vibrations of $\mathrm{OH}$ and $\mathrm{NH}_{2}$ groups. The band with the maximum at $2919 \mathrm{~cm}^{-1}$ is coming from stretching vibrations from $\mathrm{CH}$ groups. The group of bands ranging from 1649 to $893 \mathrm{~cm}^{-1}$ is characteristic for chitosan and includes: acetamide groups $\left(1649 \mathrm{~cm}^{-1}\right)$, glucopyranose rings and glycosidic bonds. As a result of MWassisted synthesis some significant changes in polymer FT-IR spectrum can be observed proving crosslinking process occurrence. Also an effect of microwave irradiation resulting in oxidation of hydroxymethyl groups can be noticed. In the spectrum of $\mathrm{J} 7$ sample (Figure $4 \mathrm{~b}$ ) wide band ranging from 3670 to $2670 \mathrm{~cm}^{-1}$ with the maximum at 3433 and $2911 \mathrm{~cm}^{-1}$ is typical for free carboxyl groups. The high intensity of this band indicates a significant number of $\mathrm{COOH}$ groups in the hydrogel. The band with maximum at $1714 \mathrm{~cm}^{-1}$ can be assigned to ester bonds. Small shift of this band may occur due to hydrogen bonds formation. High intensity bands with maximum at 1560 and $1394 \mathrm{~cm}^{-1}$ suggest more favored amide bonds formation during crosslinking reaction. In the FT-IR spectrum of J7 sample after sterilization with water vapor no significant changes are observed. Nevertheless, interesting band with high intensity at $1072 \mathrm{~cm}^{-1}$ can be noticed and associated with slight biomaterial thermal degradation during which hydrolysis of amide and ester bonds could occur resulting in formation of additional free hydroxyl groups in glucopyranose ring. It can be stated that sterilization process with the application of water vapor did not bring any significant changes in the chemical structure of the chitosan hydrogel. Thus it can be assumed that biomaterial maintained its properties after sterilization and did not undergo degradation process. As a result of sterilization with ethylene oxide some significant changes in the spectrum can be observed (Figure 4d). A band with very high intensity at $3443 \mathrm{~cm}^{-1}$ can be noticed, which is characteristic for hydroxyl groups. During chemical sterilization process with $\mathrm{C}_{2} \mathrm{H}_{4} \mathrm{O}$ oxirane ring opening occurred what resulted in ether bonds formation between hydroxyl groups from the hydrogel and sterilizing agent (Figure 6). Taking under consideration the intensity of new bands it may be assumed that biomaterial could change its chemical structure. The formation of new functional groups on the hydrogels may affect cells anchorage, as well as interactions with cell membranes which can cause cytotoxic effect. FT-IR spectra of samples J3, J4 and J5 were of very high similarity with no significant differences.

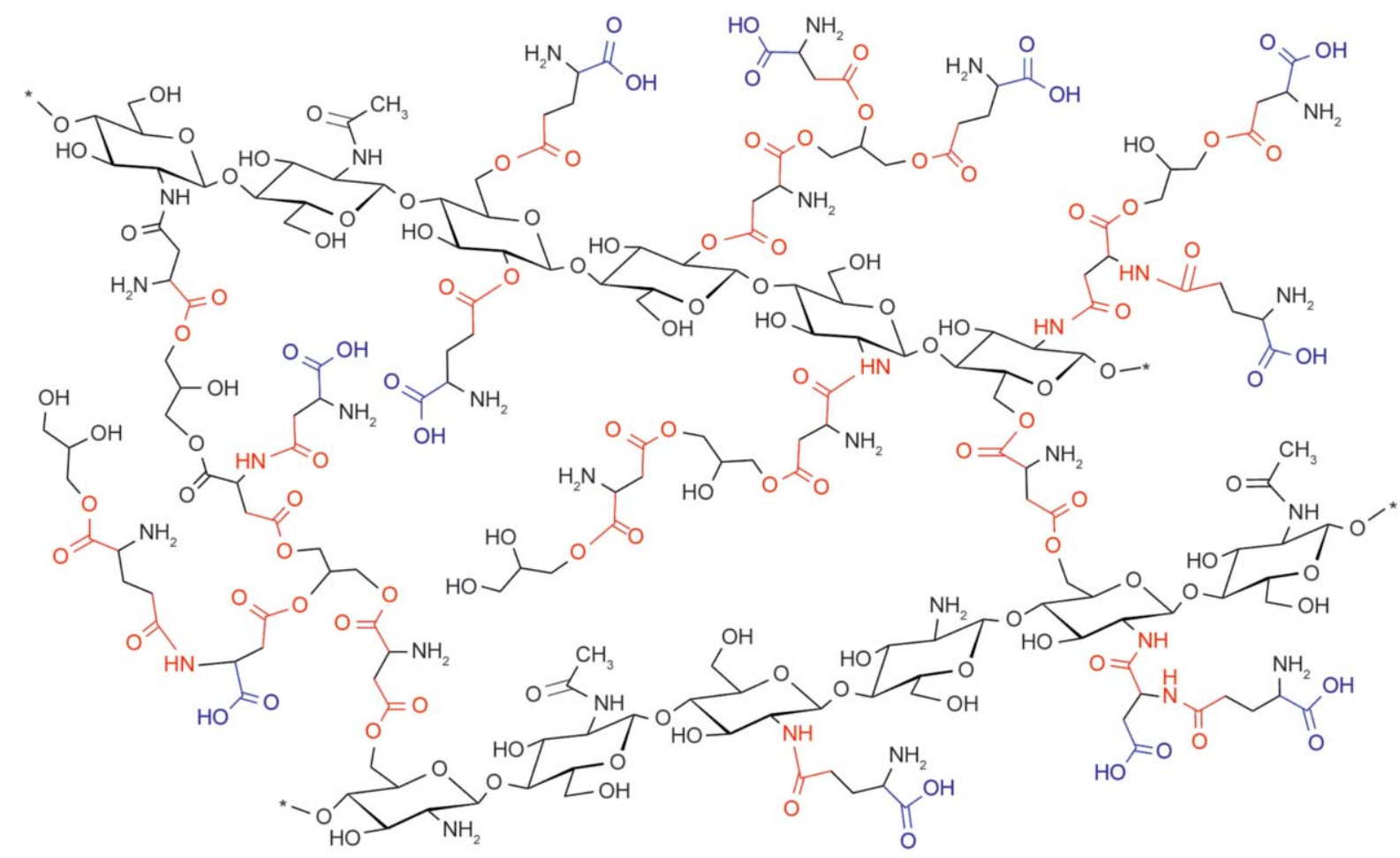

Figure 5. Proposed chemical structure of the J7 hydrogel scaffold 


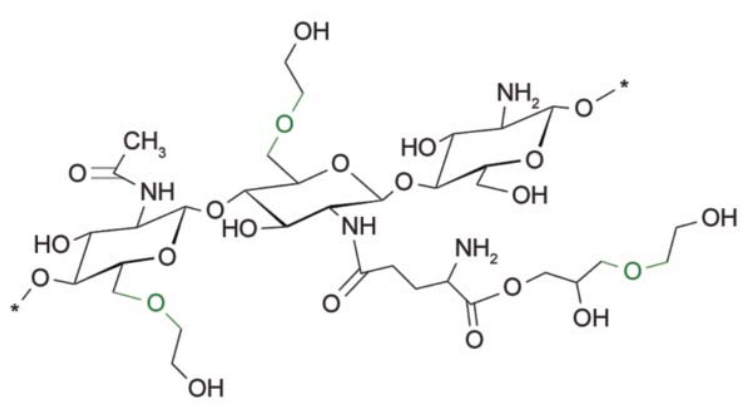

Figure 6. Proposed chemical structure of the J7 hydrogel scaffold after sterilization with ethylene oxide

Figure 5 presents proposed chemical structure of the crosslinked chitosan using Asp, Glu and glycerol. Macromolecules of the biopolymer are bound due to the formation of amide and esters of aspartic and glutamic acid with glycerol creating bridges between $\mathrm{N}$-acetylaminoglucose and aminoglucose mers. Application of negatively charged aminoacids enabled formation of amide and ester bonds resulting in three-dimensional structure obtainment. Pure chi- tosan is rich in free amino groups which are responsible for positive charge of the biopolymer surface. Too high amount of $\mathrm{NH}_{2}$ groups may lead to too strong interactions between material and cell membrane. Modification of chitosan which resulted in carboxyl and hydroxyl group formation may prevent cell membrane channels blockage thanks to repulsive forces.

\subsection{Evaluation of medium stability in culture nutrients}

$\mathrm{J} 7$ medium absorbs the largest amount of culture medium, however, it does not dissolve in the medium for the entire duration of the culture. Scaffolds J3, J4 and J5 absorbed similar amounts of culture medium, however, these J4 and J5 hydrogels crumble when subjected to mechanical treatment, which results in a larger number of small pieces getting into the medium causing visual turbidity in the samples. The turbidity was confirmed using the turbidimetric method (Figure 7). Moreover, it appears that the
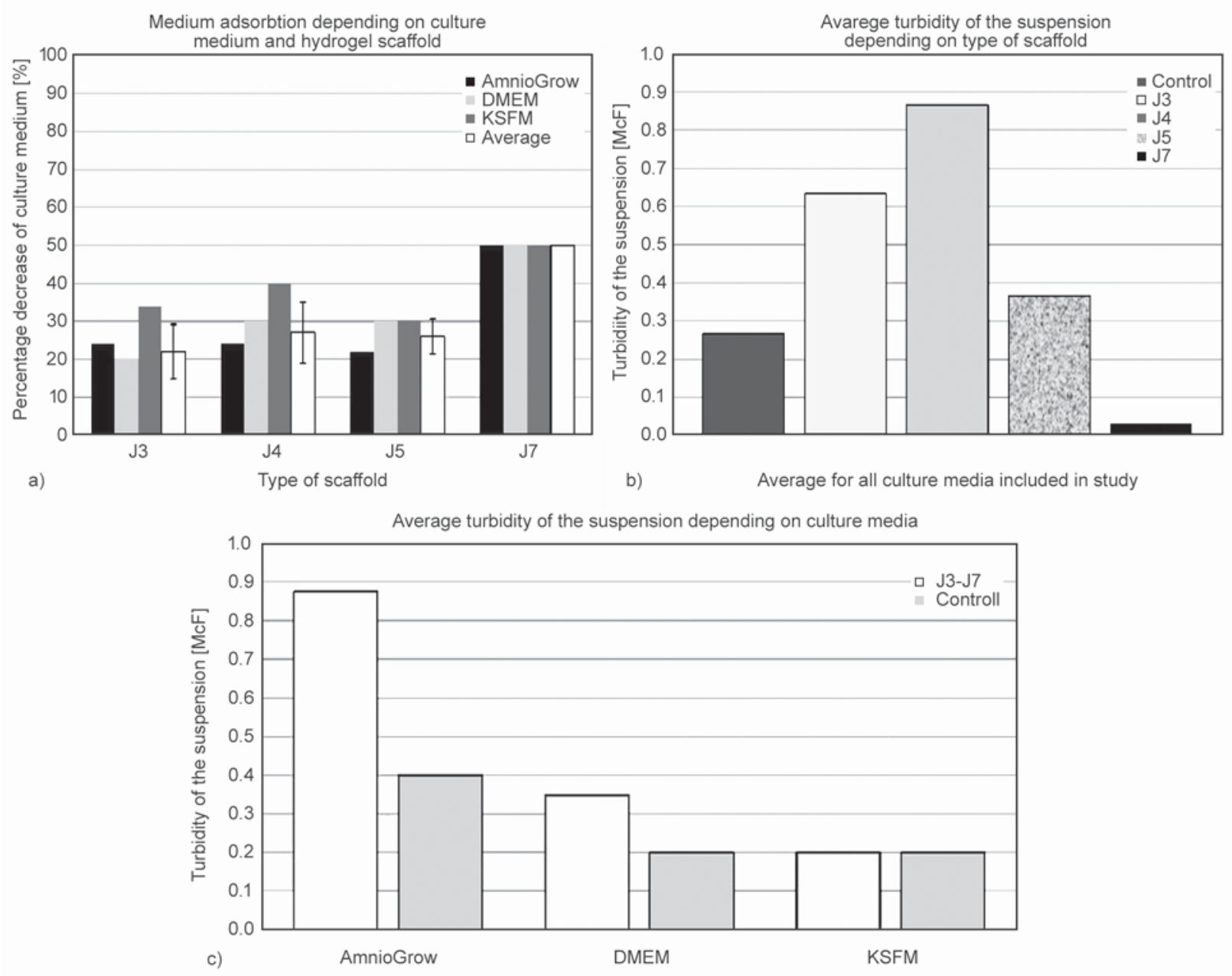

Figure 7. a) Percentage of the amount of culture nutrient absorbed by the medium, b) turbidimetrically assessed turbidity of the suspension in relation to the hydrogel scaffold type and c) types of media 
samples dissolve better in the AmnioGrow medium than in the other ones (DMEM and KDFM).

\subsection{Evaluation of sterility of media in the production process}

The samples before and after sterilisation were not colonised by either bacteria or fungi regardless of the medium.

Chitosan is known of its antibacterial and antifungal properties yet this type of bioactivity is highly correlated with average molar mass. It is proved that chitosan of high average molar mass cannot pass across the cell membrane. Therefore, it deposits over the bacteria cell membrane and blocks the nutrient transport. On the other hand, biopolymer of very low average molar mass may penetrate through cell membrane of the bacteria and bind with its genetic material. Moreover, some extra antibacterial effect is observed in case of Gram $(+)$ bacteria which have cell membrane rich in negatively charged groups (carboxyl, phosphate). Chitosan rich in $\mathrm{NH}_{3}{ }^{+}$groups may be strongly attracted to the cell membrane having negative effect on membrane integrity and surface permeability [21]. Hydrogel scaffolds were prepared from chitosan of medium average molar mass $(410000 \mathrm{~g} / \mathrm{mol})$ what suggests that obtained biomaterials should not possess antimicrobial effect.

Table 6. Microbiological growth of bacteria on the analysed media

\begin{tabular}{|c|c|c|c|}
\hline & AmnioGrow & DMEM & KSFM \\
\hline J3 & - & - & - \\
\hline J4 & - & - & - \\
\hline J5 & - & - & - \\
\hline J7 & - & - & - \\
\hline
\end{tabular}

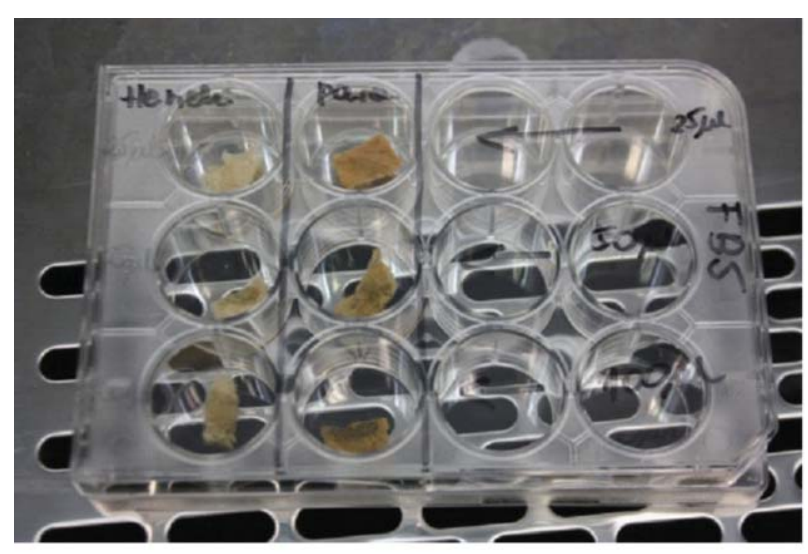

a)
Nevertheless, results presented in Table 6 proves that obtained hydrogels have antibacterial and antifungal activity, which could be enhanced by microwave irradiation application. Chemical modification of the chitosan performed to increase its durability in most cases is associated with the loss of antimicrobial properties of the biomaterial. Proposed chitosan modification pathway enables to maintain this special favor of chitin derivative.

\subsection{Standardisation of attaching media to the bottom of the bottle}

The samples attached themselves after 10 minutes regardless of the amount of FBS used what can be observed in Figure 8 (the second picture shows a twelve-hole plate upside down).

\subsection{Impact on population doubling in stem cell culture}

After $24 \mathrm{~h}$, the material did not cause contact inhibition of cell growth, however, all the samples were suspended in the medium. Moreover, it was observed that the pieces of medium that got detached and settled at the bottom of the plate also did not inhibit growth but, on the contrary, stimulated growth of the plated cells (Figure 9a, 9b). The media settled at the bottom of the dishes. After 7 days, the medium was drawn out and the number of cells attached to the medium was evaluated through the analysis of population doubling (Figure 9c). The samples sterilised with steam could be manipulated, while the samples sterilised with ethylene oxide disintegrated into small pieces at an attempt of transfer. Negative influence of ethylene oxide on the medium was also noted as affecting population doubling in a culture.

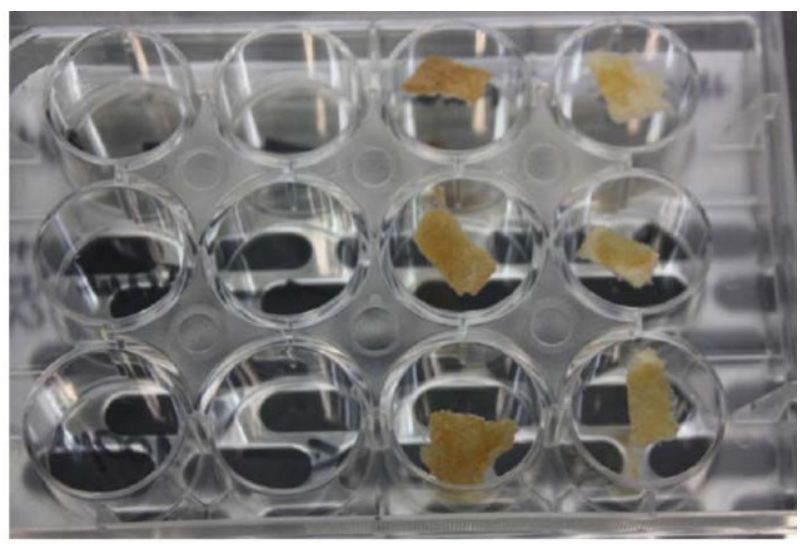

b)

Figure 8. a) Samples J7 after sterilisation attached to the medium using various amounts of foetal bovine serum. b) A twelvehole plate upside down - samples do not detach themselves from the bottom. 


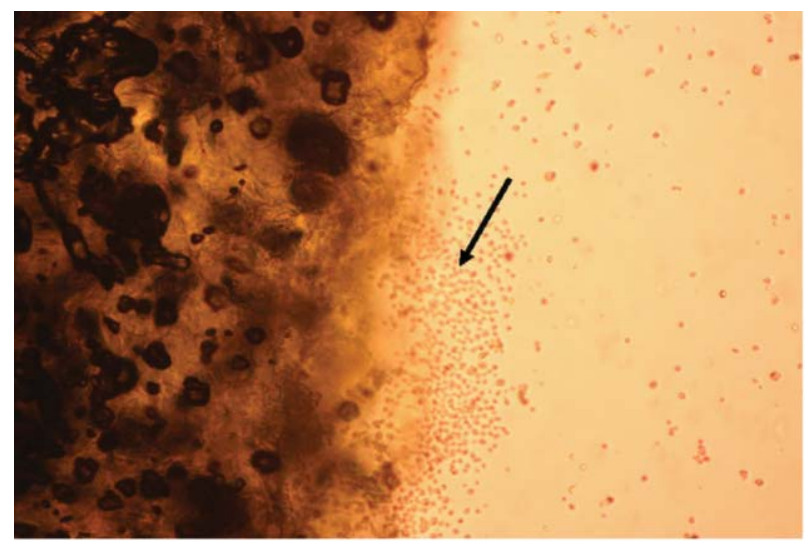

a)

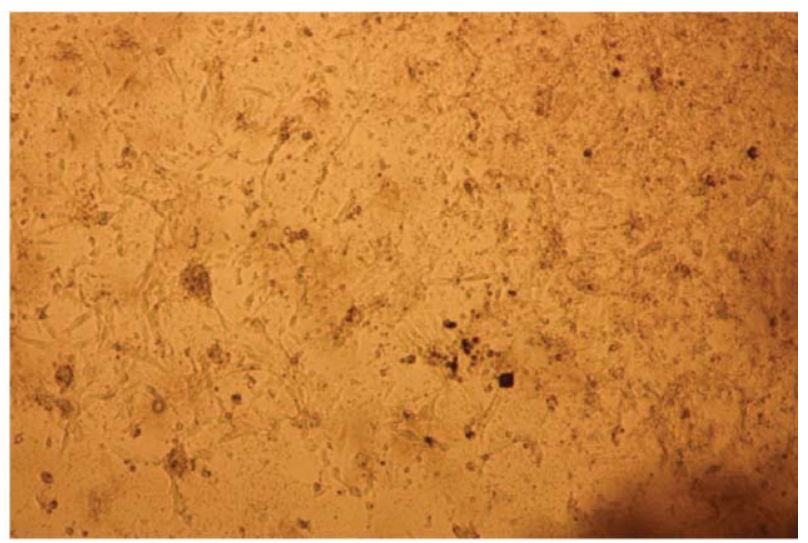

b)

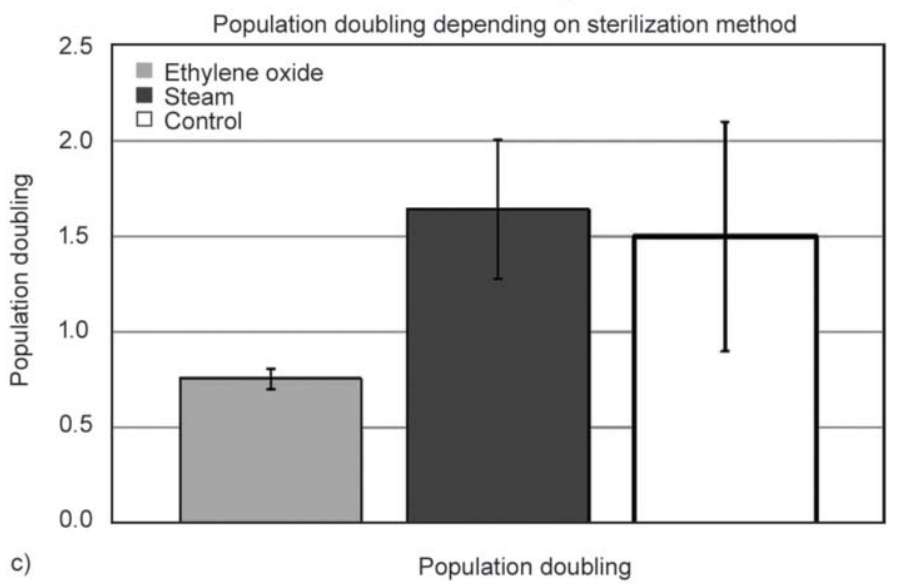

Figure 9. a) visible medium and plated cells, b) high cell growth by the small pieces settled at the bottom, $40 \times$ magnification. c) Population doubling depending on the sterilisation method

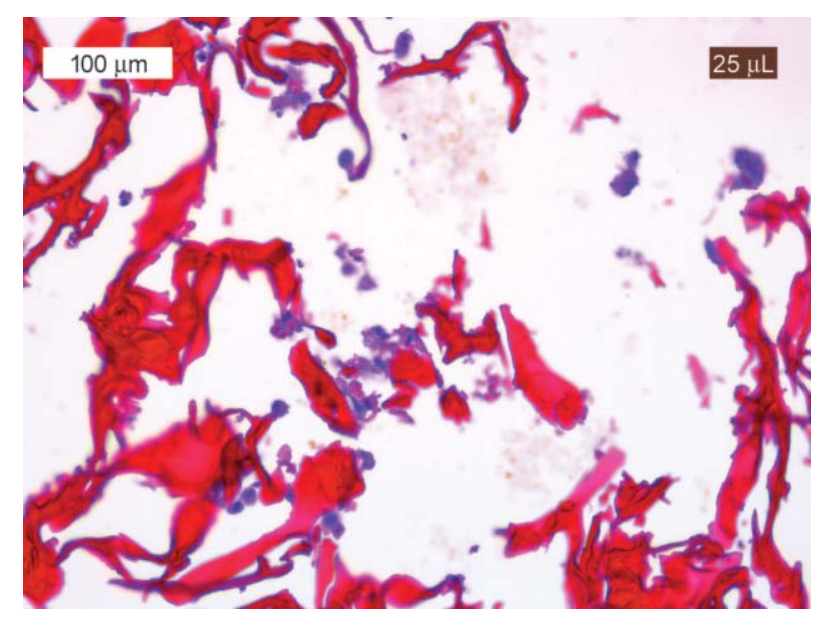

Figure 10. Visible blue-purple stained cells and red stained matrix fibres

\section{Plating the cultured stem cells on the media}

Histopathological analysis revealed the presence of cells on chitosan media sterilised by either ethylene oxide or steam (Figure 10).

\section{Conclusions}

In the present study a new type of chitosan-based hydrogel scaffolds was developed. Biopolymer crosslinking with the application of two aminoacids resulted in the formation of durable and bioactive biomaterials. The use of microwave radiation enabled rapid and successful materials preparation without application of toxic reagents. Synthesis method which meets the principles of Green Chemistry can be considered as waste-free. Obtained hydrogels were investigated over their applicability as biomaterials for MSC culture and delivery. Despite chemical modification prepared biomaterials maintained antiseptic properties of the raw chitosan. Proven porous structure enabled gases and nutrients delivery, as well as MSC anchorage.

The chitosan scaffolds presented in this study are suitable for cell culture because they can be sterilised, retain their characteristics in a culture incubator and do not cause contact growth inhibition. What 
is more, histopathological examination proved that cells that had been plated on scaffolds, although a small number of cells encapsulated themselves, remained in clusters in the hydrogel structure. The scaffolds can be easily fixed to the bottom of a culture vessel which simplifies the process of cell plating and retains the correct hydrogel orientation. However, it seems that steam sterilisation is better because ethylene oxide negatively affects both the hydrogel itself and proliferation of cells incubated with biomaterial. Further studies should consider radiation sterilisation as a method of choice in sterilisation of human transplants [14].

\section{References}

[1] Anjum F., Carroll A., Young S. A., Flynn L. E., Amsden B. G.: Tough, semisynthetic hydrogels for adipose derived stem cell delivery for chondral defect repair. Macromolecular Bioscience, 17, 1600373/1-1600373/13 (2017). https://doi.org/10.1002/mabi.201600373

[2] Kitala D., Kawecki M., Klama-Baryła A., Łabuś W., Glik J., Kraut M., Misiuga M., Nowak M.: The isolation and production of the ready-to-use product (the amniotic stem cell culture) in accordance with good manufacturing practice regulations. Stem Cells and Development, 6, 694-707 (2017).

https://doi.org/10.1089/scd.2016.0198

[3] Li F., Truong V. X., Thissen H., Frith J. E., Forsythe J. S.: Microfluidic encapsulation of human mesenchymal stem cells for articular cartilage tissue regeneration. ACS Applied Materials and Interfaces, 9, 8589-8601 (2017). https://doi.org/10.1021/acsami.7b00728

[4] Kim E. Y., Lee K-B., Kim M. K.: The potential of mesenchymal stem cells derived from amniotic membrane and amniotic fluid for neuronal regenerative therapy. BMB Reports, 47, 135-140 (2014).

https://doi.org/10.5483/BMBRep.2014.47.3.289

[5] Tsou Y-H., Khoneisser J., Huang P-C., Xu X.: Hydrogel as a bioactive material to regulate stem cell fate. Bioactive Materials, 1, 39-55 (2016).

https://doi.org/10.1016/j.bioactmat.2016.05.001

[6] Bendtsen S. T., Quinnell S. P., Wei M.: Development of a novel alginate-polyvinyl alcohol-hydroxyapatite hydrogel for 3D bioprinting bone tissue engineered scaffolds. Journal of Biomedical Materials Research Part A, 105, 1457-1468 (2017).

https://doi.org/10.1002/jbm.a.36036

[7] Placzek M. R., Chung I-M., Macedo H. M., Ismail S., Mortera Blanco T., Lim M., Cha J. M., Fauzi I., Kang Y., Yeo D. C., Ma C. Y. J., Polak J. M., Panoskaltsis N., Mantalaris A.: Stem cell bioprocessing: Fundamentals and principles. Journal of the Royal Society Interface, 6, 209-232 (2009).

https://doi.org/10.1098/rsif.2008.0442
[8] Ceccaldi C., Assaad E., Hui E., Buccionyte M., Adoungotchodo A., Lerouge S.: Optimization of injectable thermosensitive scaffolds with enhanced mechanical properties for cell therapy. Macromolecular Bioscience, 1600435/1-1600435/10 (2017).

https://doi.org/10.1002/mabi.201600435

[9] Heo D. N., Castro N. J., Lee S-J., Noh H., Zhu W., Zhang L. G.: Enhanced bone tissue regeneration using a 3D printed microstructure incorporated with a hybrid nano hydrogel. Nanoscale, 9, 5055-5062 (2017).

https://doi.org/10.1039/c6nr09652b

[10] Nicodemus G. D., Bryant S. J.: Cell encapsulation in biodegradable hydrogels for tissue engineering applications. Tissue Engineering Part B: Reviews, 14, 149-165 (2008). https://doi.org/10.1089/ten.teb.2007.0332

[11] Kim I-Y., Choi H., Kim K. K.: Improved survival of anchorage-dependent cells in core-shell hydrogel microcapsules via co-encapsulation with cell-friendly microspheres. Journal of Microencapsulation, 34, 57-62 (2017). https://doi.org/10.1080/02652048.2017.1284275

[12] Hassan W., Dong Y., Wang W.: Encapsulation and 3D culture of human adipose-derived stem cells in an insitu crosslinked hybrid hydrogel composed of PEGbased hyperbranched copolymer and hyaluronic acid. Stem Cell Research and Therapy, 4, 32/1-32/11 (2013). https://doi.org/10.1186/scrt182

[13] Sun L., Liu Z., Wang L., Cun D., Tong H. H., Yan R., Chen X., Wang R., Zheng Y.: Enhanced topical penetration, system exposure and anti-psoriasis activity of two particle-sized, curcumin-loaded PLGA nanoparticles in hydrogel. Journal of Controlled Release, 254, 44-54 (2017).

https://doi.org/10.1016/j.jconrel.2017.03.385

[14] Kitala D., Klama-Baryła A., Kawecki M., Kraut M., Łabuś W., Glik J., Ples M., Tomanek E., Nowak M.: Infections in the tissue material and their impact on the loss of transplants in the laboratory of in vitro cell and tissue culture with tissue bank in the years 2011-2015. Cell and Tissue Banking, 18, 61-68 (2017).

https://doi.org/10.1007/s10561-016-9597-y

[15] LogithKumar R., KeshavNarvan A., Dhivya S., Chavla A., Saravanan S., Selvamurugan N.: A review of chitosan and its derivatives in bone tissue engineering. Carbohydrate Polymers, 151, 172-188 (2016).

https://doi.org/10.1016/j.carbpol.2016.05.049

[16] Croisier F., Jérôme C.: Chitosan-based biomaterials for tissue engineering. European Polymer Journal, 49, 780792 (2013). https://doi.org/10.1016/j.eurpolymj.2012.12.009

[17] Lin J. H., Wen S. P., Chung H. Y., Chen W. C., Lou C. W.: Effects of 1-ethyl-3-(3-dimethylaminopropyl) carbodiimide cross-linking duration on the structure of chitosan/gelatin composite bone scaffolds. Applied Mechanics and Materials, 457-458, 44-48 (2014). https://doi.org/10.4028/www.scientific.net/AMM.457-458.44 
[18] Muzzarelli R., Mehtedi M., Bottegoni C., Aquili A., Gigante A.: Genipin-crosslinked chitosan gels and scaffolds for tissue engineering and regeneration of cartilage and bone. Marine Drugs, 13, 7314-7338 (2015). https://doi.org/10.3390/md13127068

[19] Muzzarelli R. A. A.: Genipin-crosslinked chitosan hydrogels as biomedical and pharmaceutical aids. Carbohydrate Polymers, 77, 1-9 (2009).

https://doi.org/10.1016/j.carbpol.2009.01.016
[20] de Oliviera Pedro R., Schmitt C. C., Neumann M. G.: Syntheses and characterization of amphiphilic quaternary ammonium chitosan derivatives. Carbohydrate Polymers, 147, 97-103 (2016). https://doi.org/10.1016/j.carbpol.2016.03.083

[21] Behera S. S., Das U., Kumar A., Bissoyi A., Singh A. $\mathrm{K}$.: Chitosan/ $/ \mathrm{TiO}_{2}$ composite membrane improves proliferation and survival of L929 fibroblast cells: Application in wound dressing and skin regeneration. International Journal of Biological Macromolecules, 98, 329-340 (2017).

https://doi.org/10.1016/j.ijbiomac.2017.02.017 